Original Research Paper

\title{
A Routing Optimization Based on Cross-Layer Design for Wireless Multimedia Sensor Networks (WMSNs)
}

\author{
${ }^{1,2}$ Emansa Hasri Putra, ${ }^{1}$ Risanuri Hidayat, ${ }^{1}$ Widyawan and ${ }^{1}$ Wayan Mustika \\ ${ }^{I}$ Department of Electrical Engineering and Information Technology, \\ Universitas Gadjah Mada, Indonesia \\ ${ }^{2}$ Department of Electrical Engineering, Politeknik Caltex Riau, Indonesia
}

Article history

Received: 22-05-2017

Revised: $13-10-2017$

Accepted: 18-10-2017

Corresponding Author: Emansa Hasri Putra, Politeknik Caltex Riau, Pekanbaru, Indonesia Email: emansa@pcr.ac.id

\begin{abstract}
Multimedia applications are routed from source nodes via neighboring nodes to a sink in Wireless Multimedia Sensor Networks (WMSNs). However, the existing routing protocols in the WMSNs do not fulfill the quality of service (QoS) constraint yet for multimedia transmission. The WMSNs have specific characteristics such as multi-hop way, limited node capability and varying bandwidth, which can affect the quality of service of multimedia applications during the routing process. The best quality of service (QoS) is required by the multimedia applications consisting of high throughput, low end-to-end delay and low packet drop rate. A routing optimization based on the cross-layer design is proposed for multimedia transmission in the WMSNs. The cross-layer design utilizes H.264/Scalable Video Coding (SVC) and IEEE 802.11e Enhanced Distributed Channel Access (EDCA) and has been implemented in Network Simulator 2 (NS2) simulation. The NS2 simulation indicates that the H.264/SVC video application has an average delay of $0.02 \mathrm{sec}$. Thus, the routing optimization has accomplished to deliver multimedia application successfully via Wireless Multimedia Sensor Network (WMSN).
\end{abstract}

Keywords: Routing Optimization, Cross-Layer Design, Wireless Multimedia Sensor Networks

\section{Introduction}

Nodes must be able to work together to transmit data from the source node via neighboring nodes to the sink in the WMSNs. Then the data from the neighboring nodes are forwarded multi-hop up to the sink. Thus, the WMSNs has advantages regarding easy and inexpensive network deployment, reliability because of many alternative routes through neighboring nodes and a wide range of multi-hop networks (Akyildiz et al., 2007).

Multimedia applications have specific characteristics such as requiring tight due dates, high throughput, unwavering quality, low delay and high data rate during transmission in the wireless network. Furthermore, the WMSNs are constrained by the sensor nodes capabilities that only have limited power, memory and computation. Thus, multimedia applications will undergo a process of degradation regarding throughput, delay and packet loss when the multimedia packets are routed through multiple nodes to a sink in the WMSNs (Misra et al., 2008; Ehsan and Hamdaoui, 2012).
In previous works, some researches related to routing process and multimedia transmission have been done in changeable wireless networks. The routing protocol has been categorized into four designs, consisting of Network Organization, Interaction Model, Topology and Trustworthy Routing in the Wireless Sensor Networks (WSNs) (Pantazis et al., 2013; Al-Karaki and Kamal, 2004). Ad hoc On-Demand Distance Vector (AODV) and Destination-Sequenced Distance-Vector Routing (DSDV) protocols have been investigated in a mobile ad-hoc network (Yadav and Yadav, 2008). Dynamic Source Routing (DSR) and Ad Hoc On-Demand Distance Vector Routing (AODV) have been discussed in an ad-hoc wireless network (Perkins, 2001). Ant Colony Optimization protocols have been proposed in an ad-hoc wireless networks (Caro et al., 2005; Ducatelle, 2007) and wireless sensor networks (Saleem, 2009; Putra, 2016). A cross-layer optimization has been utilized for Scalable Video Coding (SVC) video transmission in a wireless network (Foh, 2007; Li et al., 2011). A cross-layer method considers the scheduling in 
MAC and the routing protocol for real-time transmission in the WSNs (Alwan and Agarwal, 2013). A cross-layer approach has been utilized for video transmission based on H.264/SVC and Enhanced Distributed Channel Access (EDCA) in the WMSNs (Putra, 2015).

The existing routing protocols only focus on the network layer and do not consider the data link and physical layers in the wireless ad hoc networks and the wireless sensor networks. As a consequence, when the routing protocols transmit multimedia traffic in the wireless network, multimedia traffic will experience degradation regarding the quality of service (QoS). The throughput, delay and packet loss can turn influenced by varying wireless bandwidth channels conditions all the time because of fading and interference factors.

In this paper, a routing optimization is proposed to give guaranteed bandwidth (QoS) for multimedia applications in the Wireless Multimedia Sensor Networks (WMSNs). The routing optimization based on a cross-layer method determines best-selected links between sensor nodes and a sink. Since wireless sensor networks have varying bandwidth channels, a routing protocol must adapt to the varying bandwidth conditions. The cross-layer design method makes cooperation between the application, network and data link layers to provide an ideal setting for the transmission of multimedia packets. Thus, the multimedia packets when routed through WMSNs get the first priority for the routing process at the network layer. Furthermore, video coding techniques based on H.264/SVC standard is utilized in the application layer to create multimedia applications to adapt to changing wireless environments. Therefore, the multimedia application will get guaranteed bandwidth or Quality of Service (QoS) when routed via multi-hop manner in WMSNs.

The remainder of this paper is established like this. The Wireless Multimedia Sensor Network (WMSN) is discussed in Section II, Cross-Layer Design method is discussed in Section III, Section IV describes Simulation Model and Section V justifies Simulation Results. Last, The Conclusion is shown in Section VI.

\section{Wireless Multimedia Sensor Network}

Wireless Multimedia Sensor Networks (WMSNs) is a sensor network consisting of several sensor nodes that have multimedia equipment such as multiple cameras, microphones and scalar sensors. The results of sensor nodes readings will be delivered via the wireless network and shown on a server.

Figure 1 indicates a concept of the WMSN for three sensor systems with diverse purposes. A single-level network of different sensor nodes captures videos, audio and scalar data from the environment and sends the data to the center node. The center node collects and executes complete processing. Then, the processing results are delivered to the server via multi-hop manner.

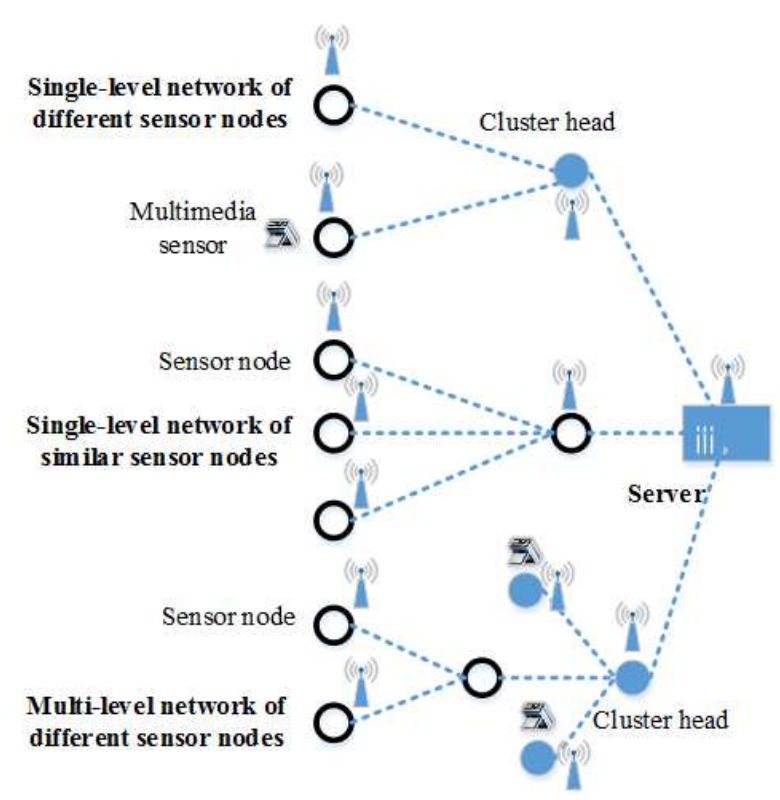

Fig. 1. A concept of Wireless Multimedia Sensor Networks (WMSNs)

A single-level network of similar sensor nodes captures similar data from the environment. Then the data is forwarded to neighboring nodes via multi-hop manner. The final destination of the data transmission is the server for processing the data. A multi-level network of different sensor nodes divides and processes audio, videos and scalar data from the environment into different layers. The lower level is responsible for scalar sensors with low capabilities. Higher level is accountable for audio and video data with high power and resources (Akyildiz et al., 2007).

\section{Cross-Layer Design}

Cross-layer design is a new approach to optimize the existing architecture of Transmission Control Protocol/Internet Protocol (TCP/IP) through finding and using the best standard of layers of TCP/IP. Each layer of the TCP/IP protocol has the duty and the service independently of one another and between adjacent layers do not communicate directly. The cross-layer design makes it possible to cooperate and communicate between the layers to find an optimization. The optimization is needed for adaptation to the wireless environment and supports QoS constraint for video applications.

The cross-layer design can be divided into three key concepts which consist of (Ksentini et al., 2006; Qian and Ya-Qin 2008):

1. Parameter Abstraction. The information needs to be collected from the application, data link and 
physical layers through a process of abstraction. Specific parameters of the existing protocol layers are selected into the parameters that are likely implemented by the cross-layer optimizer

2. Cross-Layer Optimization. The parameters obtained from the parameter abstraction is then optimized to achieve a certain purpose

3. Decisions Distribution. Results of cross-layer optimization are then redistributed to the corresponding layers

A cross-layer design method can be described mathematically using a dynamic programming model (Bradley, 1977) as shown in Fig. 2. A dynamic programming optimization is an approach that transforms a complex problem into a specific sequence or parts of smaller problems. By using the mathematical model of the dynamic programming, the cross-layer design method is divided into four stages $(n)$, starting from the rear, $n=1$. Thus, the mathematical model of the dynamic programming for the network is:

$\mathrm{v}_{4}\left(\mathrm{~s}_{4}\right)=\operatorname{Max}\left[\begin{array}{l}\mathrm{v}_{4}\left(\mathrm{~d}_{4}, \mathrm{~s}_{4}\right)+\mathrm{f}_{3}\left(\mathrm{~d}_{3}, \mathrm{~s}_{3}\right) \\ +\mathrm{f}_{2}\left(\mathrm{~d}_{2}, \mathrm{~s}_{2}\right)+\mathrm{f}_{1}\left(\mathrm{~d}_{1}, \mathrm{~s}_{1}\right)\end{array}\right]$

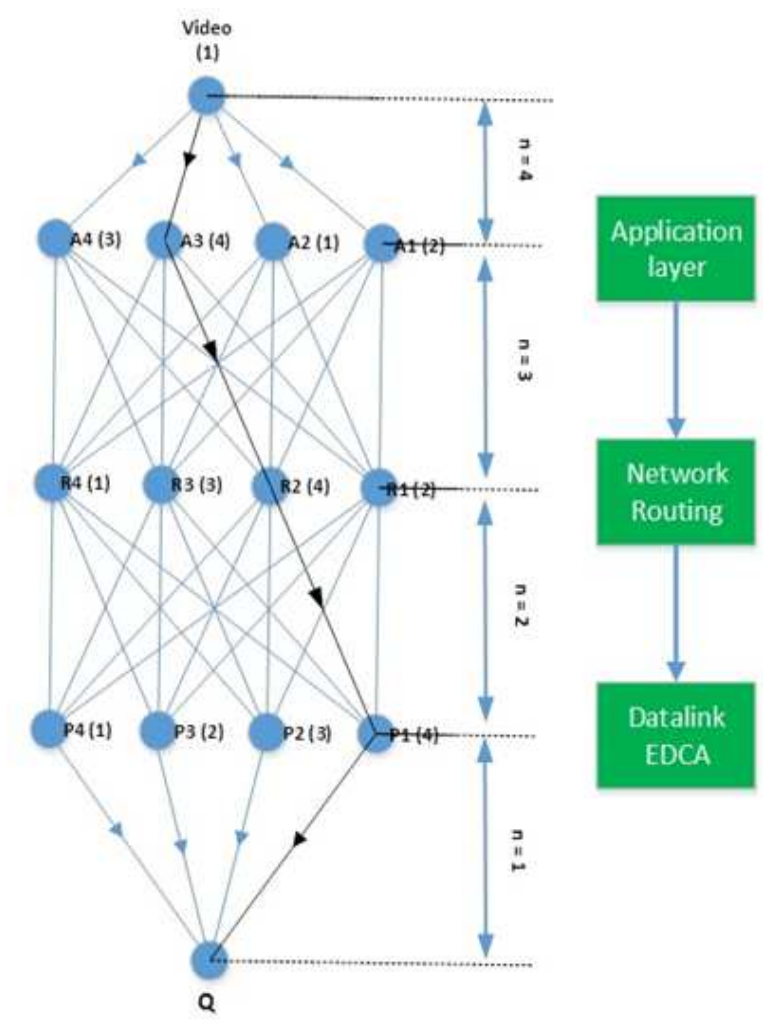

Fig. 2. A dynamic programming model for the cross-layer design method
In cross-layer design, the optimization process is conducted on three layers of TCP/IP, namely Application (A), Network (R) and Datalink (P). At the Application layer, there are four video coding techniques with different qualities (Q), namely A1 (2), A2 (1), A3 (4) and A4 (1). At the Network layer, there is four routing technique that produces different qualities (Q), namely R1 (2), R2 (4), R3 (3) and R4 (1). At Datalink layer, there are four different priorities of IEEE 802.11e Enhanced Distributed Channel Access (EDCA) scheme with different qualities (Q), namely P1 (4), P2 (3), P3 (2) and P4 (1). The value of the number in brackets indicates the value of the quality $(\mathrm{Q})$, where the greater value indicates better quality $(\mathrm{Q})$.

Completion of optimization problems using dynamic programming approach to the method of Cross-Layer Design is carried forward (forward recursive equation) and consists of four stages of the decision. The math of the problem is based on the equations 1 and is explained using the tables for each decision stage.

In the first stage, there are four options available: P1 (4), P2 (3), P3 (2), and P4 (1). The optimization result from stage 1 is P1 (4) because it has a better quality (Q) that is 4 , as shown in Table 1 . In the second stage, there are four options available: R1 (2), R2 (4), R3 (3) and $\mathrm{R} 4$ (1). The optimization result from stage 2 is $\mathrm{R} 2$ (4) because it has a better quality (Q) that is 4 , as shown in Table 2. In the third stage, there are four options available: A1 (2), A2 (1), A3 (4), and A4 (1).

Table 1. The Results of The First Stage.

\begin{tabular}{llll}
\hline$S_{1} \backslash d_{1}$ & $f_{n}$ & $\operatorname{Max}$ & $v_{1}\left(s_{1}\right)$ \\
\hline P1 & 4 & 4 & $\mathrm{Q}$ \\
$\mathrm{P} 2$ & 3 & 3 & $\mathrm{Q}$ \\
$\mathrm{P} 3$ & 2 & 2 & $\mathrm{Q}$ \\
$\mathrm{P} 4$ & 1 & 1 & $\mathrm{Q}$ \\
\hline
\end{tabular}

Table 2. The results of the second stage

\begin{tabular}{lllllll}
\hline$S_{2} \backslash d_{2}$ & $\mathrm{P} 1$ & $\mathrm{P} 2$ & $\mathrm{P} 3$ & $\mathrm{P} 4$ & $\operatorname{Max}$ & $v_{2}\left(s_{2}\right)$ \\
\hline $\mathrm{R} 1$ & $2+4=6$ & $2+3=5$ & $2+2=4$ & $2+1=3$ & 6 & $\mathrm{P} 1$ \\
$\mathrm{R} 2$ & $4+4=8$ & $4+3=7$ & $4+2=6$ & $4+1=5$ & 8 & $\mathrm{P} 1$ \\
$\mathrm{R} 3$ & $3+4=7$ & $3+3=6$ & $3+2=5$ & $3+1=4$ & 7 & $\mathrm{P} 1$ \\
$\mathrm{R} 4$ & $1+4=5$ & $1+3=4$ & $1+2=3$ & $1+1=2$ & 5 & $\mathrm{P} 1$ \\
\hline
\end{tabular}

Table 3. The Results of The Third Stage

\begin{tabular}{lllllll}
\hline$S_{3} \backslash d_{3}$ & $\mathrm{R} 1$ & $\mathrm{R} 2$ & $\mathrm{R} 3$ & $\mathrm{R} 4$ & $\operatorname{Max}$ & $v_{3}\left(s_{3}\right)$ \\
\hline $\mathrm{A} 1$ & $2+6=8$ & $2+8=10$ & $2+7=9$ & $2+5=7$ & 10 & $\mathrm{R} 2$ \\
$\mathrm{~A} 2$ & $1+6=7$ & $1+8=9$ & $1+7=8$ & $1+5=6$ & 9 & $\mathrm{R} 2$ \\
$\mathrm{~A} 3$ & $4+6=10$ & $4+8=12$ & $4+7=11$ & $4+5=9$ & 12 & $\mathrm{R} 2$ \\
$\mathrm{~A} 4$ & $3+6=9$ & $3+8=11$ & $3+7=10$ & $3+5=8$ & 11 & $\mathrm{R} 2$ \\
\hline
\end{tabular}

Table 4. The results of the fourth stage

\begin{tabular}{lllllll}
\hline$S_{4} \backslash d_{4}$ & $\mathrm{~A} 1$ & $\mathrm{~A} 2$ & $\mathrm{~A} 3$ & $\mathrm{~A} 4$ & $\operatorname{Max}$ & $v_{4}\left(s_{4}\right)$ \\
\hline Video & 10 & 9 & 12 & 11 & 12 & $\mathrm{~A} 3$ \\
\hline
\end{tabular}


The optimization result from stage 3 is A3 (4) because it has a better quality $(\mathrm{Q})$ of 4 , as shown in Table 3 . In the fourth stage, the video application chooses A3 (4), as shown in Table 4.

The result of optimization shows to get the video transmission with the highest quality (Q) by selecting the $\mathrm{A} 3 \rightarrow \mathrm{R} 2 \rightarrow \mathrm{P} 1 \rightarrow \mathrm{Q}$ from the Application layer, Network and Datalink.

\section{Simulation Model}

To examine the proposed optimization, NS2 simulation (Ke, 2012) was conducted as shown in Fig. 3 including two ways. The first, the sensor node 1 sends four important data stream with 512 bytes packet size to the sensor node 3 with speeds of $600 \mathrm{kbps}$ in WMSN. In the network layer, the Ad hoc On-Demand Distance Vector (AODV) and the Destination-Sequenced Distance Vector Routing (DSDV) protocols are utilized interchangeably. Then throughput, packets end-to-end delay and packet drop rate is calculated throughout the simulation.
The second, the sensor node 1 sends the "Foreman" H.264/SVC video (Schwarz et al., 2007) to the sensor node 5 in WMSN. Furthermore three data stream with 512 bytes packet size are also sent in WMSN with speeds of $600 \mathrm{kbps}$. In the network layer, the Ad hoc On-Demand Distance Vector (AODV) and the Dynamic Source Routing (DSR) protocols are utilized interchangeably. Then throughput, packets end-to-end delay and packet drop rate is calculated throughout the simulation.

To optimize the routing process for multimedia transmission in the WMSN, the procedure of cross-layer design of the Open Systems Interconnection (OSI) layers is conducted. The process of parameter abstraction collected important data from the three layers consisting of application, network and data link layers. Furthermore the process of cross-layer optimization is conducted to fulfill the requirements of Quality of Service (QoS) for multimedia transmission. Then the process of decisions distribution is conducted to deliver the results of the data optimization back into the application, network and data link layer.

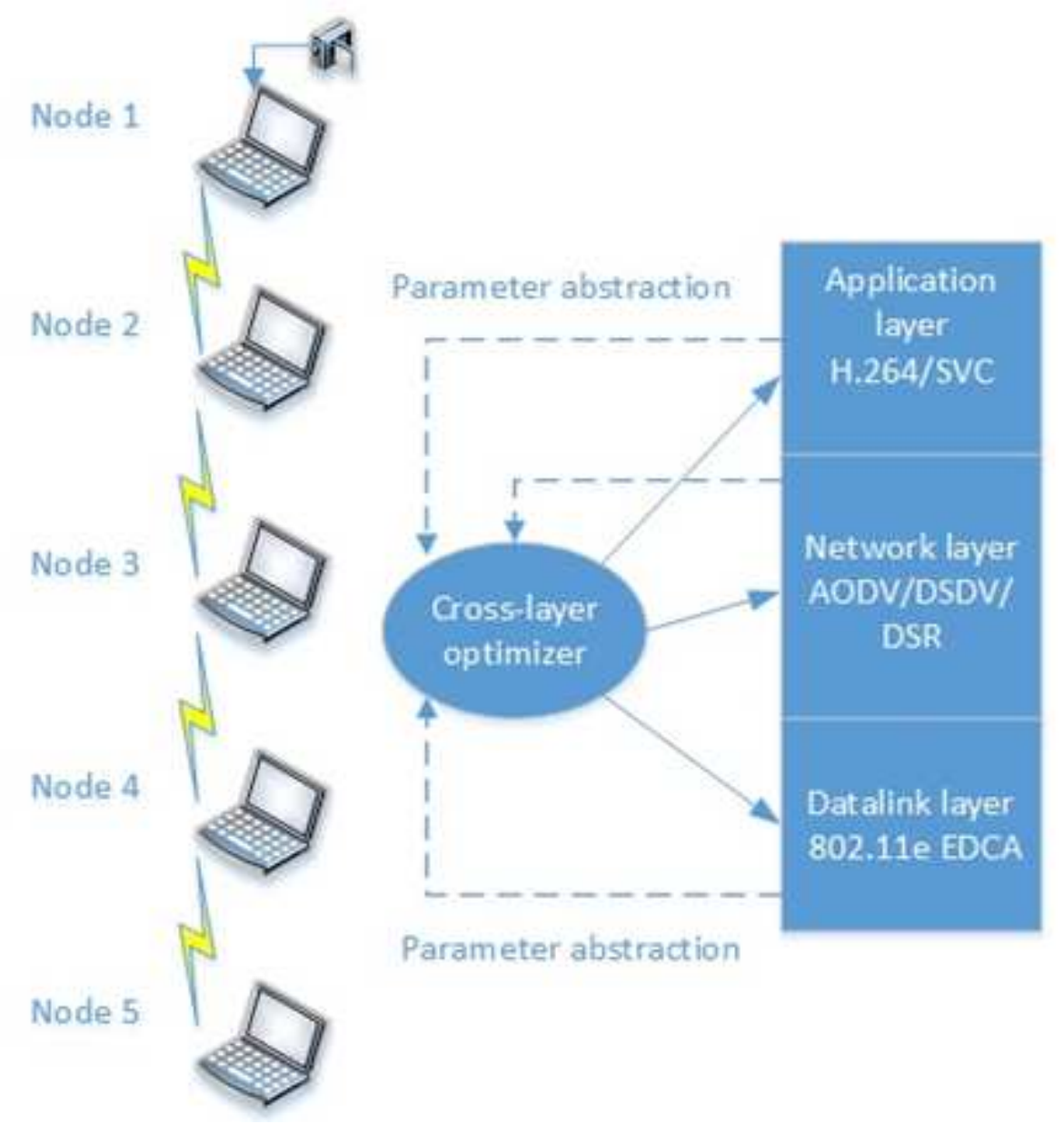

Fig. 3. The simulation model 
The proposed cross-layer design utilized H.264/SVC as a video coding standard at the application layer because the video coding standard has supported many existing device technologies and multimedia applications. Furthermore, the video coding standard also supports low bit rate for multimedia transmission in WMSN. The proposed cross-layer design utilized the Ad hoc On-Demand Distance Vector (AODV), the Destination-Sequenced Distance Vector Routing (DSDV) and the Dynamic Source Routing (DSR) as routing protocols at the network layer. The routing protocols represent R1, R2 and R3 in dynamic programming approach as explained in Section 3. The proposed cross-layer design utilized the IEEE 802.11e Enhanced Distributed Channel Access (EDCA) as a new channel access method for the Media Access Control (MAC) layer at the data link layer. The EDCA has supported four priority classes $(\mathrm{P})$, starting from the highest priority to the lowest priority. The priority classes $(\mathrm{P})$ are intended for voice traffic, video, best effort and background correspondingly. These priorities of EDCA represent P1, P2 and P3 in dynamic programming approach.

\section{Simulation Results}

The throughput for four data stream is indicated in Fig. 4 via WMSN where AODV and DSDV protocols are utilized interchangeably. Based on the EDCA scheme in the datalink layer, the first important data stream gets the first priority to access existing bandwidth, the second important data stream gets the second priority, the third important data stream gets the third priority and the fourth important data stream gets the fourth priority.

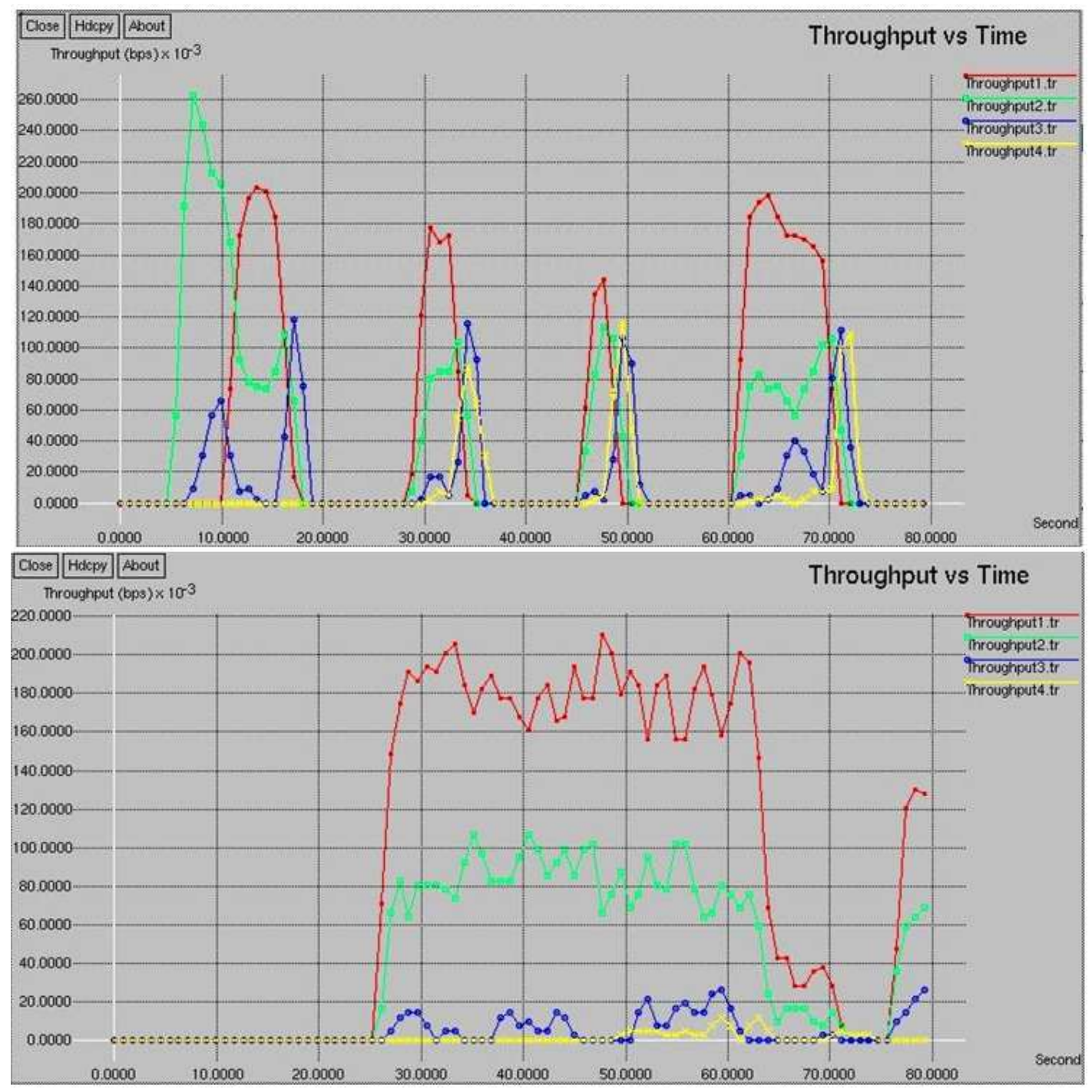

Fig. 4. The throughput for four important data stream through WMSN where AODV (above) and DSDV (below) protocols are utilized 

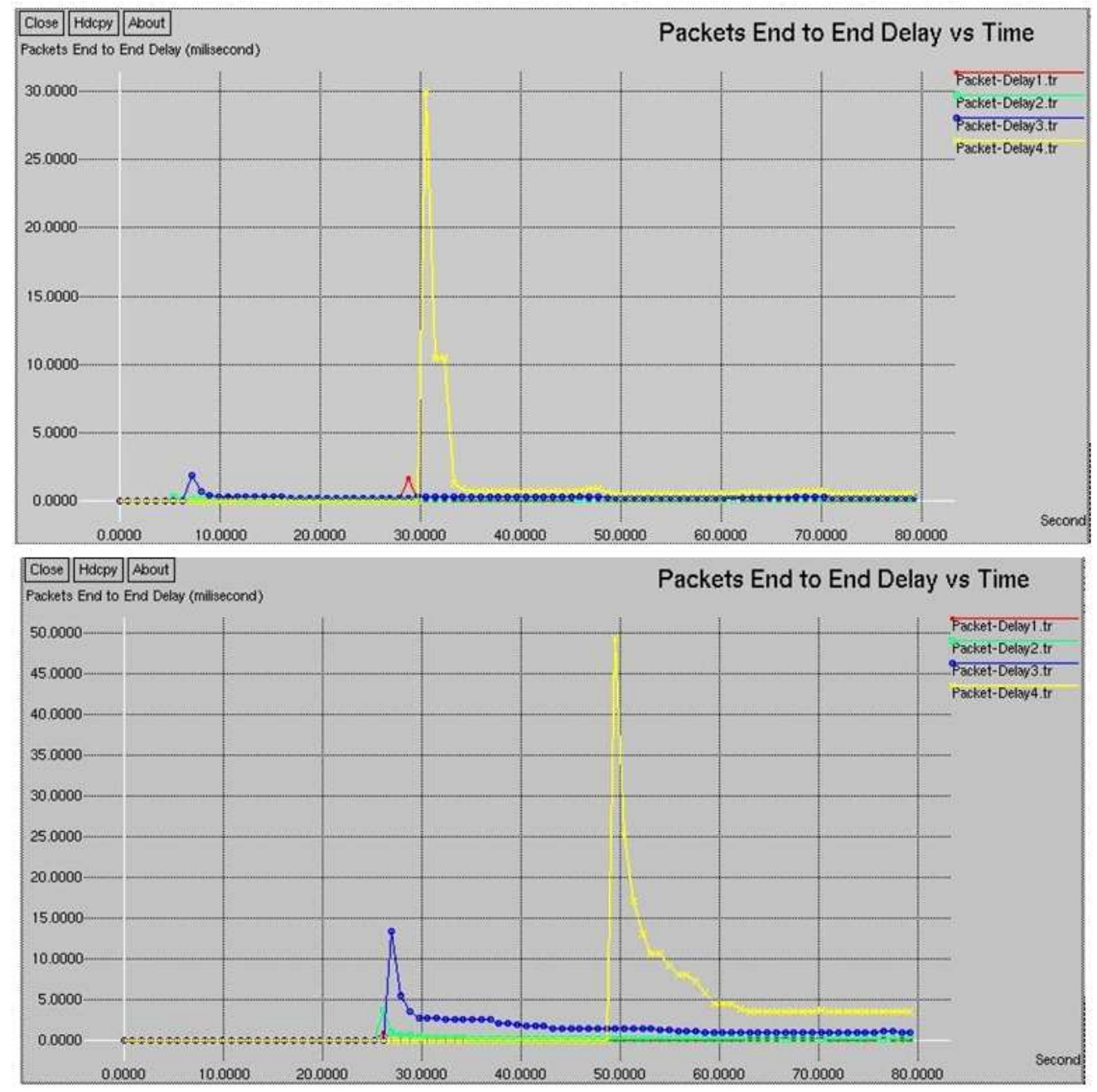

Fig. 5. The rate of packets end-to-end delay for four important data stream through WMSN where AODV (above) and DSDV (below) protocols are utilized

The throughput of the first priority data is more stable (indicated by the red graph) than other priority data when utilizing DSDV protocol at the network layer. Whereas, when utilizing AODV protocol, the throughput of all the priority data tends to be unstable. DSDV protocol utilizes table-driven routing and DSDV routing information is always updated in routing tables. Otherwise, AODV protocol utilizes on-demand routing protocol. Due to the sensor nodes are all in a fixed position, multimedia packets are routed faster and have a lower delay through WMSN when utilizing DSDV protocol. Thus, the routing optimization based on crosslayer design provides better throughput for the first important data stream when DSDV and EDCA protocols are utilized in the proposed cross-layer design. The rate of packets end-to-end delay for four data stream is indicated in Fig. 5 via WMSN where AODV and DSDV protocols are utilized interchangeably. The rate of packets end-toend delay remains very low for the first important data stream (indicated by the red graph) when DSDV and EDCA protocols are utilized in the model of cross-layer design. DSDV protocol utilizes pre-determined routes while AODV protocol finds routes when needed. Thus, AODV protocol has a higher delay than DSDV protocol.

The rate of packets loss or drop for four data stream is indicated in Fig. 6 via WMSN where AODV and DSDV protocols are utilized interchangeably. The first important data stream get high packets drop rate when AODV and EDCA protocols are utilized in the simulation. Due to AODV protocols utilize an ondemand approach to finding routes. Thus, multimedia packets experience high packet drop rate when routed in WMSN. The first important data stream has low packets drop rate when DSDV and EDCA protocols are utilized in the simulation. It indicates that DSDV has better quality for routing $(\mathrm{R})$ and EDCA provides the first priority $(\mathrm{P})$ for scheduling in data link layer. 


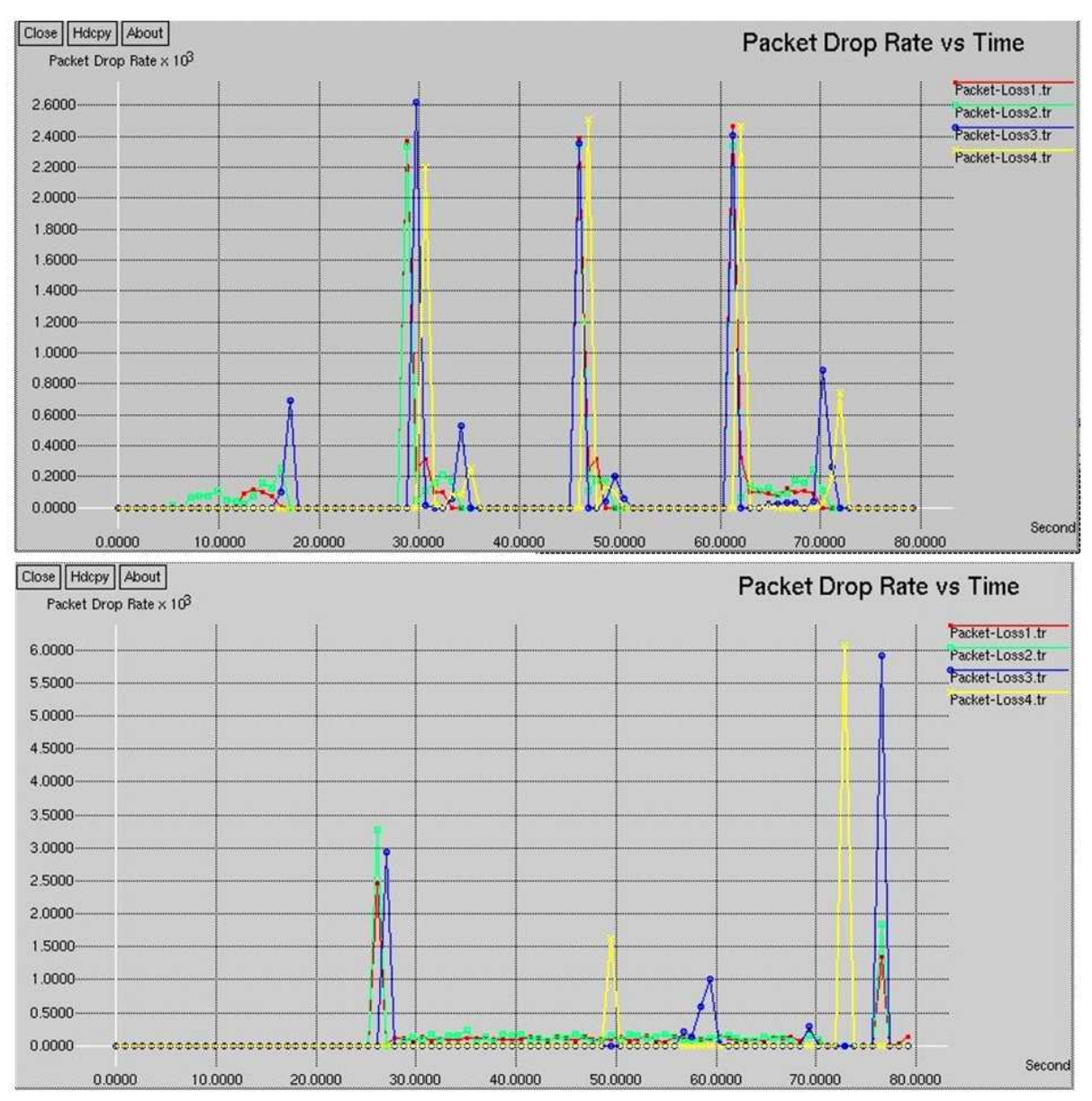

Fig. 6. The rate of packets loss or drop four important data stream through WMSN where AODV (above) and DSDV (below) protocols are utilized

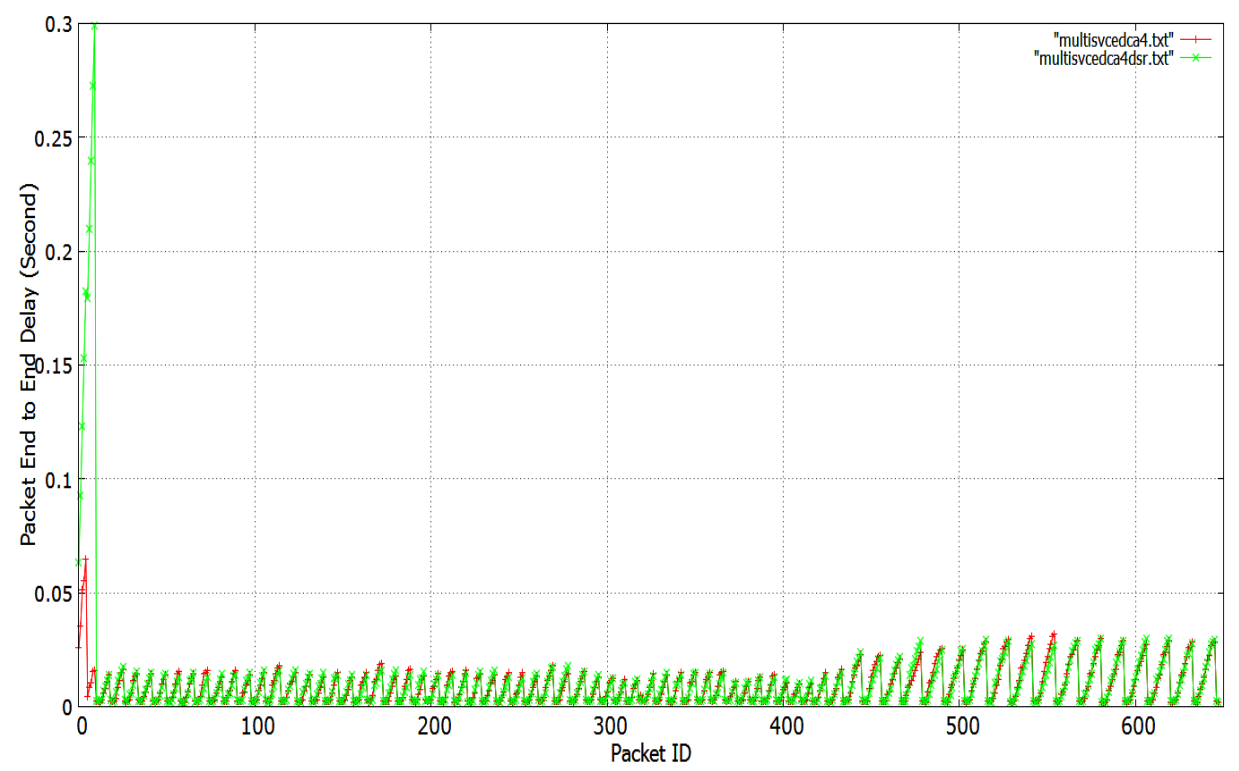

Fig. 7. The rate of packets end-to-end delay for the "Foreman" H.264/SVC video from the sensor node 1 to the sensor node 5 where AODV and DSR protocols are utilized 
The rate of packets end-to-end delay for the "Foreman" H.264/SVC video is indicated in Fig. 7. The video is delivered from the sensor node 1 to the sensor node 5 and AODV and DSR protocols are utilized interchangeably. When DSR protocol is utilized in the network layer, the "Foreman" H.264/SVC video has lower end-to-end delay (indicated by the green graph) than AODV protocol in the network layer. The "Foreman" H.264/SVC video packets have an average delay of 0.02 second. DSR protocol utilizes many alternative routes from sources to a sink. Each node in the DSR has a routing source that stores information for all these purposes.

Then each node also has an ability to learn the route to each destination in DSR. Thus, DSR has a better ability to obtain routing information than AODV. It indicates that the routing optimization based on crosslayer design provides lower delay results for the "Foreman" H.264/SVC video when DSR and EDCA protocols are utilized in the model of cross-layer design.

\section{Conclusion}

The routing optimization based on the proposed cross-layer design has been conducted successfully in the wireless multimedia sensor network (WMSN). The routing optimization has treated the first important data stream to obtain better results regarding throughput, packets end-to-end delay and packet drop rate in the WMSN. Then the "Foreman" H.264/SVC video packets have lower delay when AODV and DSR protocols are utilized in the cross-layer design. Thus, the routing optimization process based on the proposed cross-layer design can optimize the multimedia transmission in the wireless multimedia sensor networks (WMSNs).

\section{Acknowledgement}

This work was supported by Department of Electrical Engineering and Information Technology, Universitas Gadjah Mada and Department of Electrical Engineering, Politeknik Caltex Riau, Indonesia.

\section{Funding Information}

This work was supported by Politeknik Caltex Riau Dissertation Grant, Indonesia.

\section{Author's Contributions}

Emansa Hasri Putra: Proposed a routing optimization model, conducted simulations to investigate it and wrote the manuscript.

Risanuri Hidayat: Discussed and revised all paper content.

Widyawan: Analyzed how to implement the crosslayer design model for routing optimization in WMSN.

Wayan Mustika: Analyzed how to use dynamic programming approach for the cross-layer design model.

\section{Ethics}

This article is original and contains unpublished material. The corresponding author confirms that all of the other authors have read and approved the manuscript and there are no ethical issues involved.

\section{References}

Akyildiz, I.F., T. Melodia and K.R. Chowdhury, 2007. A survey on wireless multimedia sensor networks. Comput. Netw., 51: 921-960. DOI: 10.1016/j.comnet.2006.10.002

Al-Karaki, J.N. and A.E. Kamal, 2004. Routing techniques in wireless sensor networks: A survey. IEEE Wireless Communications, 11: 6-28.

DOI: $10.1109 / \mathrm{mwc} .2004 .1368893$

Alwan, H. and A. Agarwal, 2013. A cross-layer-based routing with QoS-aware scheduling for wireless sensor networks. Proceedings of the ACS International Conference on Computer Systems and Applications, May 27-30, IEEE Xplore press, Ifrane, Morocco, pp: 1-8.

DOI: 10.1109/AICCSA.2013.6616449

Bradley, H., 1977. Chapter 11 - Dynamic Programming. Applied mathematical programming, 26: 320-362. DOI: $10.2307 / 2550876$

Caro, G.D., F. Ducatelle and L.M. Gambardella, 2005. Special Issue on Self-organisation in mobile networking AntHocNet: An adaptive nature-inspired algorithm for routing in mobile ad hoc networks. Eur. Trans. Telecommunications.

DOI: $10.1002 /$ ett.1062

Ducatelle, F., 2007. Adaptive routing in ad hoc wireless multi-hop networks. Diss. Università della Svizzera Italiana.

Ehsan, S. and B. Hamdaoui, 2012. A survey on energyefficient routing techniques with QoS assurances for wireless multimedia sensor networks. IEEE Communications Surveys Tutorials, 14: 265-278. DOI: 10.1109/SURV.2011.020211.00058

Foh, C.H., 2007. Optimized cross-layer design for scalable video transmission over the IEEE 802.11e networks. IEEE Transactions Circuits Systems Video Technology, 17: 1665-1678. DOI: 10.1109/TCSVT.2007.903808

Ke, C.H., 2012. myEvalSVC: An integrated simulation framework for evaluation of H.264/SVC transmission. KSII Transactions Internet Information Syst., 6: 378-393. DOI: $10.3837 /$ tiis.2012.01.021

Ksentini, A., M. Naimi and A. Gueroui, 2006. Toward an improvement of H.264 video transmission over IEEE 802.11e through a cross-layer architecture. IEEE Communications Magazine, 44: 107-114. DOI: 10.1109/MCOM.2006.1580940 
Li, M., Z. Chen and Y.P. Tan, 2011. Cross-layer optimization for SVC video delivery over the IEEE $802.11 \mathrm{e}$ wireless networks. J. Visual Communication Image Representation, 22: 284-296. DOI: $10.1016 /$ j.jvcir.2011.01.002

Misra, S., M. Reisslein and G. Xue, 2008. A survey of multimedia streaming in wireless sensor networks. IEEE Communications Surveys Tutorials, 10: 18-39. DOI: $10.1109 /$ SURV.2008.080404

Pantazis, N.A., S.A. Nikolidakis and D.D. Vergados, 2013. Energy-efficient routing protocols in wireless sensor networks: A survey. IEEE Communications Surveys Tutorials, 15: 551-591. DOI: 10.1109/SURV.2012.062612.00084

Perkins, C.E., 2001. Performance comparison of two ondemand routing protocols for ad hoc networks. IEEE Personal Communications, 8: 16-28. DOI: 10.1109/98.904895

Putra, E.H., 2015. Cross-layer design of wireless multimedia sensor network based on IEEE 802.11e EDCA and H.264/SVC. Proceedings of the International Conference on Science in Information Technology, Oct. 27-28, IEEE Xplore press, Yogyakarta, Indonesia.

DOI: 10.1109/ICSITech.2015.7407779
Putra, E.H., 2016. A routing optimization based on ant colony for Wireless Multimedia Sensor Networks (WMSNs). Int. J. Intelligent Eng. Syst., 9: 179-184. DOI: $10.22266 /$ ijies2016.1231.19

Qian, Z. and Z. Ya-Qin, 2008. Cross-layer design for QoS support in multihop wireless networks. Proceedings IEEE, 96: 64-76. DOI: $10.1109 /$ JPROC.2007.909930

Saleem, K., 2009. Ant based self-organized routing protocol for wireless sensor networks. Int. J. Communication Networks Information Security, 1: 42-46.

Schwarz, H., D. Marpe and T. Wiegand, 2007. Overview of the scalable video coding extension of the H.264/AVC Standard. IEEE Transactions Circuits Systems Video Technol., 17: 1103-1120. DOI: 10.1109/TCSVT.2007.905532

Yadav, N.S. and R.P. Yadav, 2008. Performance comparison and analysis of table-driven and ondemand routing protocols for mobile ad-hoc networks. India, pp: 2809-2817. 